\title{
Rotor Position and Velocity Estimation of SMO Based on PLL
}

\author{
Xueliang Ren ${ }^{\mathrm{a}}$, Mingjiang Wang ${ }^{\mathrm{b}}$, Jun Chen ${ }^{\mathrm{c}}$ \\ Harbin Institute of Technology, Shenzhen 518000, China \\ arenxueliang@stu.hit.edu.cn, ${ }^{b}$ mjwang@hit.edu.cn, cjunchen.time@foxmail.com
}

Keywords: Field-oriented control, Sliding mode observer, PLL, Sensorless control

\begin{abstract}
In view of the running safety in elevator under earthquake, several damage forms of elevator are presented and its analysis model is set up. Then some earthquake protective measures for elevators are proposed. In order to inspect the seismic behavior of the elevator, the seismic simulated vibration test machine of elevator is further analyzed based on the advanced control theory in this paper. Finally, the seismic simulated vibration test machine of elevator is developed and the results show that the method can be applied to elevators. These studies laid the foundation for further exploring the elevator safety performance in earthquake environment.
\end{abstract}

\section{Introduction}

In the field-oriented control, in order to achieve a high-performance of three-phase PMSM control system, it is generally required to obtain accurate rotor position and rotor speed information. The conventional method often uses a mechanical position sensor, but the use of the mechanical sensor will increase the system cost, size and weight. And there are more stringent requirements for the use of the environment. The sensorless control technology uses a certain control algorithm to detect rotor position and velocity by detecting the relevant electrical signals in the motor windings.

Sliding mode control is a special non-linear control system. The fundamental difference between it and conventional control lies in the discontinuity of control, that is, a switching characteristic that makes the "structure" of the system change at any time. The key to the realization of this method lies in the selection of the sliding surface function and the selection of the sliding mode gain. It is necessary to ensure the speed of convergence, and also need to avoid chattering in the motor running caused by too large gain. Cause the sliding mode control does not require high accuracy of the system model and it is insensitive to parameter changes and external disturbances, so it is a very robust control method. In the three-phase PMSM control system, the method is based on the error between the given current and the feedback current, and this error is used to reconstruct the back EMF of the motor and estimate the rotor speed. In addition, the error can be used to design a sliding mode observer (SMO)

The traditional sliding mode control usually uses arc tangent function to calculate the back EMF, but it will be accompanied by high-frequency chattering in the sliding mode, so there will be highfrequency chattering in the estimated speed and position waveform, and The division operation in the arctangent function will amplify this high-frequency chatter. Therefore, the phase-locked loop (PLL) system is used to extract the position information and speed information of the rotor in this paper, and the sliding mode observer algorithm is designed on the synchronous rotary coordinate.

\section{SMO Design in Synchronous Rotary Coordinate System}

Three-phase AC motor is a multi-variable system with strong coupling, non-linearity and high order. Its mathematical model in the three-phase stationary coordinate system is quite complicated. It is very difficult to apply traditional control strategies to achieve AC speed regulation. So many papers often use vector control method to control three-phase AC motor, and use coordinate transformation. Coordinate transformation converts three-phase AC motor control to DC motor control. The mathematical model of the motor can be greatly simplified in the synchronous rotary coordinate 
system. Therefore, this paper establishes the mathematical model of the PMSM in synchronous rotary coordinate system. PMSM mathematical model in synchronous rotary coordinate system:

$$
\left\{\begin{array}{l}
u_{d}=R i_{d}+L_{d} \frac{d i_{d}}{d t}-\omega_{e} L_{q} i_{q} \\
u_{q}=R i_{q}+L_{q} \frac{d i_{q}}{d t}-\omega_{e}\left(L_{d} i_{d}+\psi_{f}\right)
\end{array}\right.
$$

\subsection{Sliding Mode Observer Design}

This section is to design the SMO, so rewrite the mathematical model of the motor

$$
\left\{\begin{array}{l}
\frac{d i_{d}}{d t}=\frac{1}{L_{d}}\left(-R i_{d}+u_{d}-\omega_{e} L_{q} i_{q}-\mathrm{E}_{d}\right) \\
\frac{d i_{q}}{d t}=\frac{1}{L_{q}}\left(-R i_{q}+u_{d}-\omega_{e} L_{d} i_{d}-\mathrm{E}_{q}\right)
\end{array}\right.
$$

Where: $\mathrm{E}_{d}=0, \mathrm{E}_{q}=\omega_{e} \psi_{f}$, they can be seen as back EMF in the $d-q$ synchronous rotary coordinate system.

In order to obtain the value of back EMF in equation (1-2), SMO can be designed as

$$
\begin{aligned}
& \left\{\begin{array}{l}
\frac{d \hat{i}_{d}}{d t}=\frac{1}{L_{d}}\left(-R \hat{i_{d}}+u_{d}-\omega_{e} L_{q} \hat{i}_{q}-V_{d}\right) \\
\frac{d \hat{i}_{q}}{d t}=\frac{1}{L_{q}}\left(-R \hat{i}_{q}+u_{d}-\omega_{e} L_{d} \hat{i}_{d}-V_{q}\right)
\end{array}\right. \\
& \left\{\begin{array}{l}
V_{d}=k \operatorname{sgn}\left(\hat{\mathrm{i}}_{d}-\mathrm{i}_{d}\right) \\
V_{q}=k \operatorname{sgn}\left(\hat{\mathrm{i}}_{q}-\mathrm{i}_{q}\right)
\end{array}\right.
\end{aligned}
$$

Where: $\hat{\mathrm{i}}_{d} \hat{\mathrm{i}}_{q}$ are the current observed values for the stator $\mathrm{d}$-axis and q-axis, respectively; $\mathrm{k}$ is the sliding mode gain.

Equations (1-2) and (1-3) are subtracted to obtain the equation of current error system state as

$$
\left\{\begin{array}{l}
\frac{d \tilde{i_{d}}}{d t}=\frac{1}{L_{d}}\left(-R \tilde{i_{d}}+E_{d}-\omega_{e} L_{q} \tilde{i}_{q}-V_{d}\right) \\
\frac{d \tilde{i_{q}}}{d t}=\frac{1}{L_{q}}\left(-R \tilde{i_{q}}+E_{q}-\omega_{e} L_{d} \tilde{i}_{d}-V_{q}\right)
\end{array}\right.
$$

Where: $\tilde{i}_{d}=\hat{i}_{d}-i_{d}, \tilde{i}_{q}=\hat{i}_{q}-i_{q}$ are the current observation error.

The current is estimated using SMO. The sliding surface function is defined as

$$
\tilde{\boldsymbol{i}}=\left[\begin{array}{ll}
\tilde{i}_{d} & \tilde{i}_{q}
\end{array}\right]^{T}=\mathbf{0}
$$

SMO enters sliding mode when the following conditions are met:

$$
\tilde{\boldsymbol{i}}^{T} \dot{\boldsymbol{i}}<0
$$

When the sliding mode gain is large enough, the inequality (1-7) is established and the system enters the sliding mode, that is

$$
\tilde{\boldsymbol{i}}^{T}=\dot{\tilde{\boldsymbol{i}}}=\mathbf{0}
$$

So the back EMF can be calculated as follows

$$
\boldsymbol{E}=\left[\begin{array}{l}
{\left[k \operatorname{sgn}\left(\hat{\mathrm{i}}_{d}-\mathrm{i}_{d}\right)\right]_{e q}} \\
{\left[k \operatorname{sgn}\left(\hat{\mathrm{i}}_{q}-\mathbf{i}_{q}\right)\right]_{e q}}
\end{array}\right]^{T}=\left[\begin{array}{c}
0 \\
\omega_{e} \psi_{f}
\end{array}\right]
$$

Then according to the sliding mode arrival condition $\tilde{\boldsymbol{i}}^{T} \dot{\tilde{\boldsymbol{i}}}<0$, the expression of the gain $\mathrm{k}$ can be obtained: 


$$
k=n \max \left[\frac{E_{d}}{L_{d}} \operatorname{sgn}\left(\tilde{\mathrm{i}}_{d}\right)-\left(\frac{R}{L_{d}}+\frac{L_{d}}{L_{q}} \omega_{e}\right)\left|\tilde{\mathrm{i}}_{d}\right|, \frac{E_{q}}{L_{q}} \operatorname{sgn}\left(\tilde{\mathrm{i}}_{q}\right)-\left(\frac{R}{L_{q}}+\frac{L_{q}}{L_{d}} \omega_{e}\right)\left|\tilde{\mathrm{i}}_{q}\right|\right]
$$

Where: $\mathrm{n}$ is positive constant, usually $\mathrm{n}=2$ can satisfy the sliding mode arrival condition $\tilde{\boldsymbol{i}}^{T} \dot{\tilde{\boldsymbol{i}}}<0$.

\section{Rotor Position Estimation Based on PLL}

The purpose of designing the SMO is to obtain the estimated value of back EMF in the d-q axis. It can be seen from the formula (1-2) that the q-axis back EMF contains the rotor velocity information, that is, $\mathrm{E}_{q}=\omega_{e} \psi_{f}$. Thus, according to formula (1-9), the estimated value of q-axis can be obtained and the rotor speed is

$$
\hat{\omega}_{e}=\frac{V_{q}}{\psi_{f}}
$$

Although the position angle of the rotor can be obtained by integrating equation (1-11), the actual value of the magnetic flux of the permanent magnet is not a constant, which is due to various factors (such as temperature and load) during the actual operation of the motor. Therefore, the rotor position and speed estimation in this manner will deviate from the actual value, which will affect the dynamic performance of the entire system. To obtain better dynamic performance, this article uses PLL technology. It is an adaptive closed-loop system that has excellent real-time ability to track and estimate actual rotor position information. Even in some bad conditions such as unbalanced voltage phase angles and large harmonics, it has good tracking performance. Since the windings of the motor are symmetrical, it is assumed that the terminal voltage of the three-phase stator winding of the motor is

$$
\left\{\begin{array}{l}
u_{a}=u \cos \omega_{e} t \\
u_{b}=u \cos \left(\omega_{e} t-2 \pi / 3\right) \\
u_{c}=u \cos \left(\omega_{e} t+2 \pi / 3\right)
\end{array}\right.
$$

Where: $\mathrm{u}$ is the terminal voltage amplitude, let $\theta_{e}=\omega_{e} t$, and $\omega_{e}=\pi p_{n} / 30, p_{n}$ is the number of motor poles and $\mathrm{n}$ is the mechanical motor speed.

According to the transformation theory of synchronous rotary coordinates, the transformation matrix of three-phase voltage into $\mathrm{d}$ - q coordinate system is

$$
\boldsymbol{T}\left(\hat{\theta}_{e}\right)=\frac{1}{3}\left[\begin{array}{ccc}
\cos \hat{\theta}_{e} & \cos \left(\hat{\theta}_{e}-\frac{2}{3} \pi\right) & \cos \left(\hat{\theta}_{e}+\frac{2}{3} \pi\right) \\
-\sin \hat{\theta}_{e} & -\sin \left(\hat{\theta}_{e}-\frac{2}{3} \pi\right) & -\sin \left(\hat{\theta}_{e}+\frac{2}{3} \pi\right)
\end{array}\right]
$$

Where: $\hat{\theta}_{e}$ is the estimated phase angle of the output which is used by PLL technology, and $\hat{\theta}_{e}=\hat{\omega}_{e} t$

Define $\tilde{\theta}_{e}=\hat{\theta}_{e}-\theta_{e}$,it is the estimated error of the PLL, and as long as appropriate adjustments are made, the rotor position estimate can be converged to the actual rotor position.

Substituting the transformation matrix $\boldsymbol{T}\left(\hat{\theta}_{e}\right)$ into equation (1-12), taking into account neutral isolation, generally it does not include zero-sequence components. The equation in the $\mathrm{d}-\mathrm{q}$ coordinate system is:

$$
\left[\begin{array}{l}
V_{d} \\
V_{q}
\end{array}\right]=\left[\begin{array}{l}
u \sin \left(\hat{\theta}_{e}-\theta_{e}\right) \\
u \cos \left(\hat{\theta}_{e}-\theta_{e}\right)
\end{array}\right]
$$


When the PLL estimation value tracks the actual position of rotor, the error $\tilde{\theta}_{e}$ is zero. According to the definition of the synchronous rotary coordinate system $\mathrm{d}-\mathrm{q}$, there should be $V_{d r e f}=V_{d}=0$, so the closed-loop PI regulator can be constructed by formula (1-14) to obtain rotor position information. The specific implementation block diagram is shown in Figure 1.

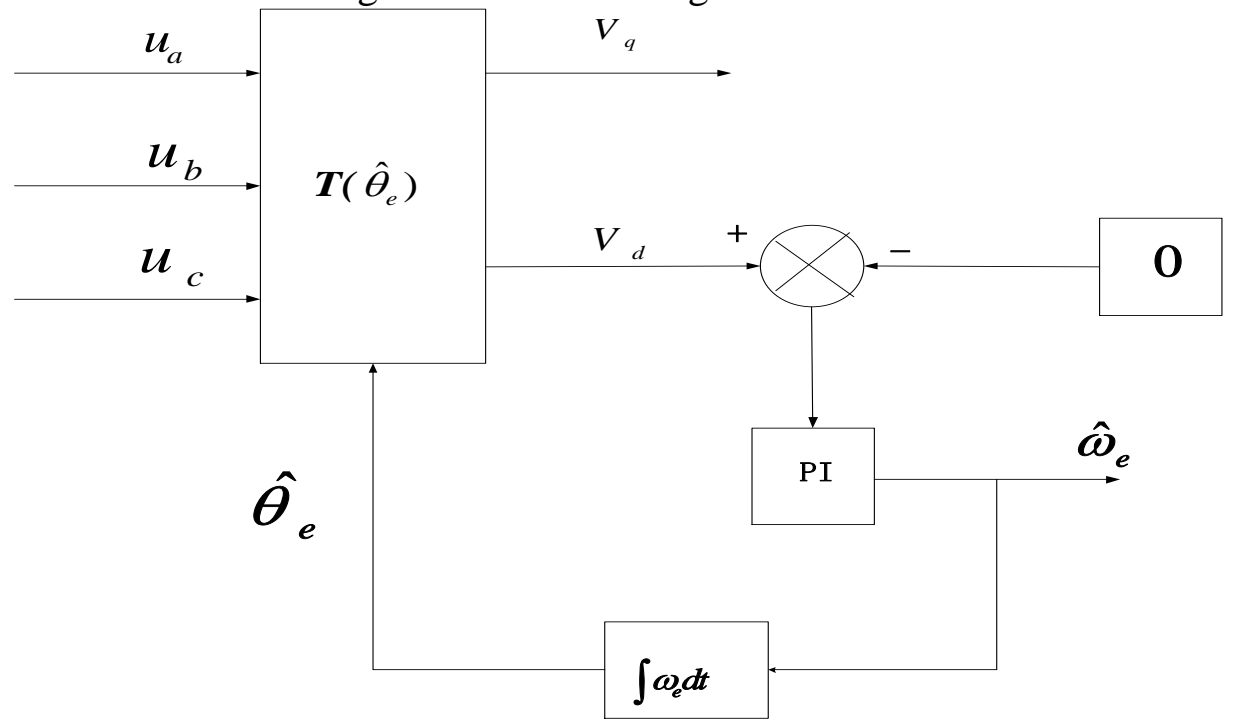

Figure 1 Block diagram of rotor position based on PI regulator

\section{Simulation}

Computer simulation is used to verify the estimation performance of the novel sliding mode control observer. MATLAB/Simulink is the simulation platform. The Simulink model of the PMSM vector control system has been constructed including the proposed sliding mode observer and the conventional sliding mode observer based on arc tangent function. And the two algorithm simulations are conducted in the same condition so that the proposed algorithm performance can be evaluated objectively. The parameters of PMSM are shown in Table1.

Table 1 PMSM simulation parameters

\begin{tabular}{|c|c|}
\hline Parameter & Value \\
\hline Speed $(w)$ & $1000[\mathrm{r} / \mathrm{min}]$ \\
\hline Stator Resistor(Rs) & $0.011[\mathrm{ohm}]$ \\
\hline Stator Inductor(Lq,Ld) & $0.0016[\mathrm{H}]$ \\
\hline Rotor Inertia(J) & $0.0008 \mathrm{Kg} . \mathrm{m} 2$ \\
\hline Poles(P) & 3 \\
\hline Flux $\left(\prod f\right)$ & $0.077[\mathrm{~Wb}]$ \\
\hline
\end{tabular}

Fig. 2 shows simulation result of sliding mode observer based on an arctangent function. It can be clearly seen from the figure that due to the existence of high-frequency chattering, the estimated and actual values of rotor position, velocity and error cannot distinguish. Due to the existence of a large number of high-frequency and large amplitude interference signals, the experimental waveforms are not smooth enough, and even the actual useful signal is submerged in high-frequency signals, which makes the experimental data difficult to record.

Fig. 3 shows simulation results using the proposed method which is a SMO based on PLL. It can be seen from the figure that there are almost no high-frequency interference signals in the experimental waveform diagrams, and the experimental waveforms are relatively smooth. The experimental observations records more accurately. In addition, the overshoot of the control system is small, and the system can quickly reach a stable state. The error also approach zero in a very short time, and the dynamic performance is good. 


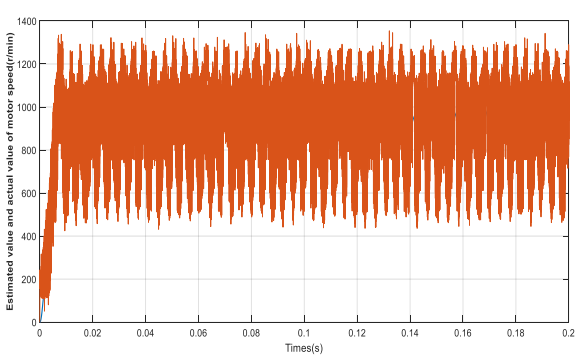

(a)

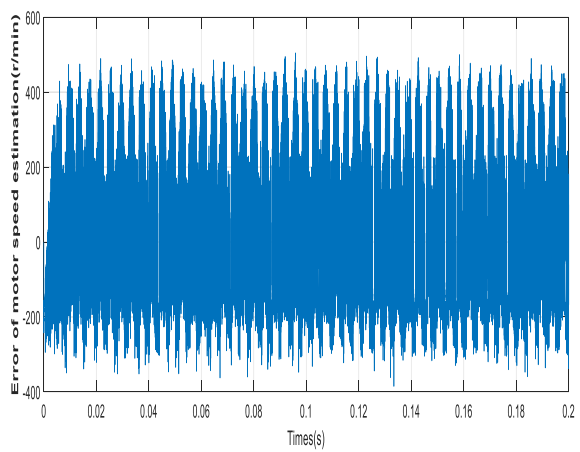

(b)

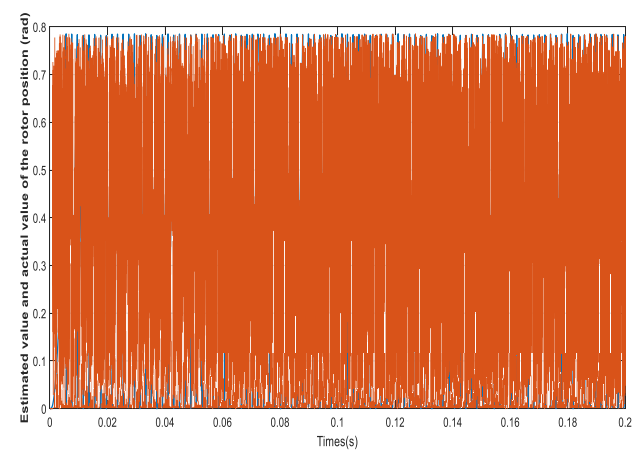

(c)

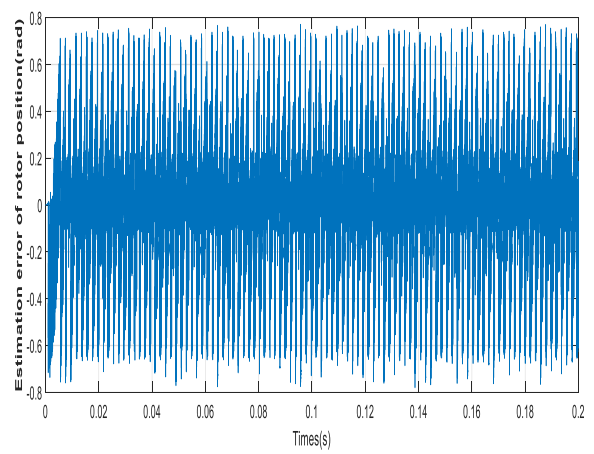

(d)

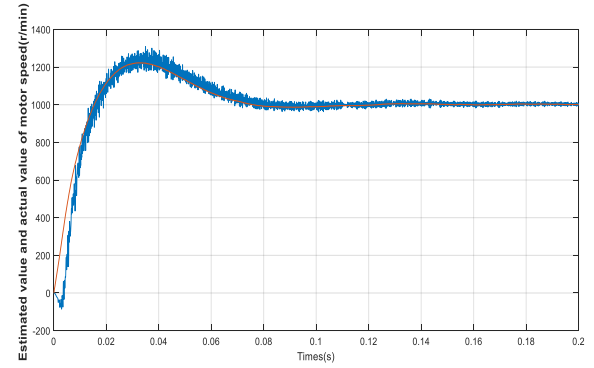

(a)

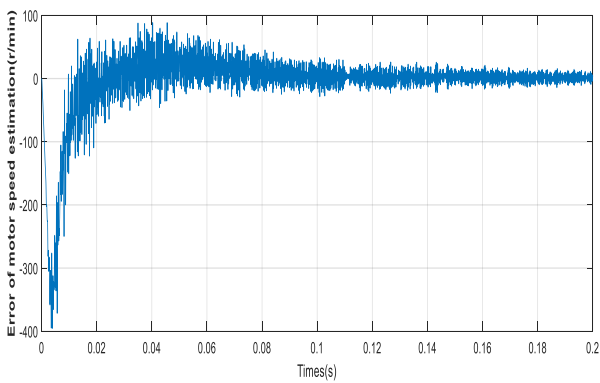

(b)

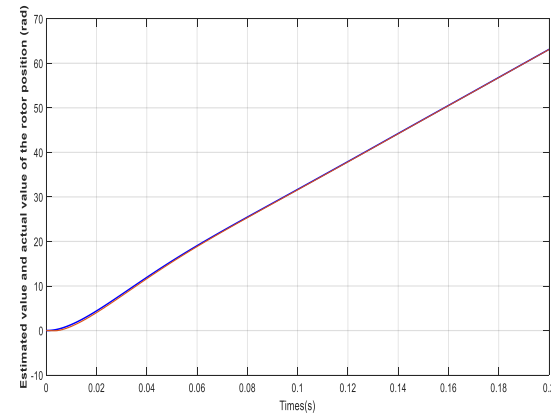

(c)

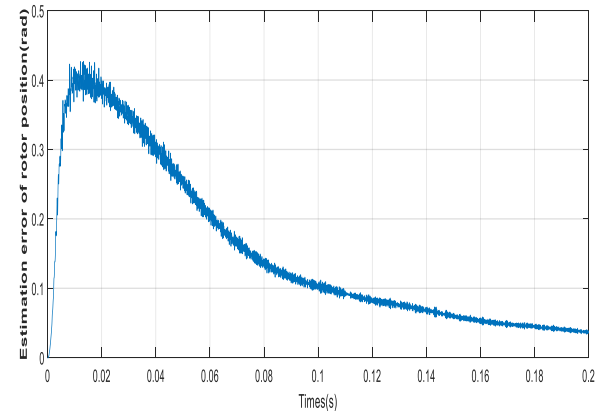

(d)

Fig.2.The results of the sliding mode based on arc tangent Fig.3.The results of the sliding mode based on PLL

\section{Conclusion}

This paper has proposed a sliding mode observer based on PLL to achieve the sensorless control for PMSM. Compared with SMO based on arctangent, the proposed method in this paper not only can effectively remove the effect of high-frequency chatter signal in the state of sliding mode, but also can significantly reduce speed estimation error and rotor position estimation error and can be quickly and accurately estimate rotor speed and position. 


\section{References}

[1]. Qi L, Jia T, Shi H. A novel sliding mode observer for PMSM sensorless vector control[J]. 2011:1646-1650.

[2]. Yang C, Ma T, Che Z, et al. An Adaptive-gain Sliding Mode Observer for Sensorless Control of Permanent Magnet Linear Synchronous Motors[J]. IEEE Access, 2017, PP (99):1-1.

[3]. Bernardes T, Gründling H A, Pinheiro H. Discrete time sliding mode observers for sensorless vector control of PMSM[C]// Xxth International Conference on Electrical Machines. IEEE, 2012:893-899.

[4]. Plestan F, Shtessel Y, Bregeault V, et al. New methodologies for adaptive sliding mode control[J]. International Journal of Control, 2010, 83(9):1907-1919.

[5]. Qiao Z, Shi T, Wang Y, et al. New Sliding-Mode Observer for Position Sensorless Control of Permanent-Magnet Synchronous Motor[J]. IEEE Transactions on Industrial Electronics, 2012, 60(2):710-719.

[6]. Ezzat M, Leon J D, Gonzalez N, et al. Sensorless speed control of permanent magnet synchronous motor by using sliding mode observer[C]// International Workshop on Variable Structure Systems. IEEE, 2010:227-232.

[7]. Xepapas S, Kaletsanos A, Xepapas F, et al. Sliding-mode observer for speed-sensorless induction motor drives[J]. Control Theory and Applications, IEE Proceedings -, 2003, 150(6):611-617.

[8]. Elbuluk M, Li C. Sliding mode observer for wide-speed sensorless control of PMSM drives[C]// Industry Applications Conference, 2003. Ias Meeting. Conference Record of the. IEEE, 2004:480-485 vol.1. 